\title{
SECRETORY IMMUNOGLOBULIN A (SIgA) TESTING IN SALIVA FROM END-STAGE PATIENTS WITH CHRONIC KIDNEY DISEASE UNDERGOING DIALYSIS TREATMENT AND IN CONTROL HEALTHY SUBJECTS
}

\author{
A. Nenova-Nogalcheva', D. Konstantinova'2, P. Pechalova ${ }^{3}$ \\ 'Department of Oral and Maxillofacial Surgery, Medical University - Varna, Bulgaria \\ ${ }^{2}$ Department of Prosthetic Dentistry, Medical University - Varna, Bulgaria \\ ${ }^{3}$ Department of Oral Surgery, Medical University - Plovdiv, Bulgaria
}

\begin{abstract}
The purpose of the present study was to measure SIgA levels in saliva in control healthy patients and in patients with end-stage chronic kidney disease (CKD) undergoing dialysis treatment. Methods: 90 participants, divided into 2 groups (Clinical Group and Control Group), had their SigA levels measured from a salivary liquid medium using radial Immunodiffusion (or Mancini method). The Clinical Group comprised 70 end-stage CKD patients on chronic dialysis treatment; the Control Group consisted of 20 clinically healthy individuals. SIgA mean values of the participants from the Clinical Group and the Control Group were $161.46 \pm 105.76$ and $69.70 \pm 25.67 \mathrm{mg} / \mathrm{l}$, respectively. Results: SlgA mean levels in female patients from the Clinical group were significantly higher compared to the mean values in male patients $(p=0.004)$. The SIgA levels in healthy participants from the Control Group did not reveal statistically significant gender-related dependencies ( $p$ $=0.699$ ). Statistical correlation between gender and SigA levels was not established for either group. However, a statistically significant positive correlation was found between the duration of the hemodialysis treatment and SlgA levels $(r=0.46, p<0.001)$. Conclusion: The results of the SIgA testing for both groups differed statistically. In the Control Group SIgA levels did not prove to be dependent on gender and age, whereas female patients with chronic renal failure exhibited significantly increased SIgA levels. The studies have outlined a tendency towards increasing SIgA levels among younger patients as well as in patients, undergoing chronic dialysis treatment for over 5 years.
\end{abstract}

Key words: salivary IgA, oral cavity, end-stage renal disease

Corresponding author: Assoc. Prof. Desislava Konstantinova, Department of Prosthetic Dentistry, Medical University, 84 "Tsar Osvoboditel" Blvd., 9000 Varna, Bulgaria, e-mail: dr.konstantinova@gmail.com

\section{INTRODUCTION}

odern scientific research uses saliva as an alternative study medium. Its advantages outweigh those of blood serum and other biological fluids. The easy collection of test material and the possibility of repeated non-invasive sample testing along with the prevention of contamination of the patient and medical staff render it suitable for the screening and diagnosis of various diseases. It is particularly favored when working with children and in difficult cases of venipuncture. 
The organic composition of saliva, among its other components, includes secretory $\lg \mathrm{A}(\mathrm{S} \lg \mathrm{A})$. Immunoglobulin $A$ is an antibody that plays a crucial role in the immune function of mucous membranes [1]. In absolute terms, between three and five grams are secreted into the intestinal lumen each day [2]. SIgA is the main immunoglobulin found in mucous secretions, including tears, saliva, sweat, colostrum and secretions from the genitourinary tract, gastrointestinal tract and the prostate. It is known that its highest concentration can be detected in the saliva of gl. parotis, where part of it enters the gland from plasma and the other part is secreted into the gland itself. Saliva contains SIgA antibodies that are natural and reactive against various saprophytic bacteria. These antibodies may control the oral microbiota by reducing the adhesion of bacteria to the oral mucosa and teeth $[3,4,5]$. It is thought that protection against bacterial etiologic agents of caries and periodontal diseases could be conferred by the induction of SIgA antibodies via the stimulation of the mucosal immune system [6].

The aim of the present study was to measure SIgA levels in saliva in control healthy subjects and in patients with end-stage chronic kidney disease (CKD) undergoing dialysis treatment.

\section{MATERIALS AND METHODS}

The enrolled patients were 90 participants from Northeastern Bulgaria, divided into 2 groups: Clinical Group and Control Group. The Clinical Group comprised of 70 end-stage CKD patients on chronic dialysis treatment: 32 male patients $(45.71 \%)$ and 38 female patients $(54.29 \%)$, with a mean age of 58.9 years (SD $=14.46$ ). The youngest participant was 32 years old, while the oldest one was an 89-year-old patient. The Control Group involved 20 clinically healthy individuals, in functional and physiological equilibrium of the body: 12 women $(60 \%)$ and 8 men $(40 \%)$, with a mean age of 22.2 years $(S D=1.94)$. The youngest participant was 19 years old and the oldest one was 27 years old. The clinical study was approved by the Research Ethics Committee at the Medical University - Varna with Protocol No. 55/ 16 June 2016.

Saliva samples were collected from patients in the morning after fasting between 10.00am and $11.00 \mathrm{am}$ to avoid the daily variability in saliva composition. An hour prior to testing the patients were asked to have their mouth rinsed with distilled water to remove food debris. Samples of unstimulated saliva were gathered within 5-minute duration of excretion directly into sterile containers using $\mathrm{Na}$ vazesh method [7]. With the purpose of avoiding precipitation of salivary mucin, the samples were frozen and subsequently transported to the clinical laboratory. Supernatants were stored at $-70^{\circ} \mathrm{C}$ until use. On the day of the assay, all samples were centrifuged for 15 minutes at $10.000 \mathrm{~g}$., at $+4^{\circ} \mathrm{C}$ until all cells and dirt were removed. Pure samples were pipetted into suitable containers. Quantitative measurement of SIgA was performed using Radial Immunodiffusion (or Mancini Method) [8]. Each IgA immuno-plate (Binding Site Group Ltd, Birmingham, B15 1QT, United Kingdom) is a ready-to-use agar gel product, containing anti-human, monospecific, high-titer, anti-lgA antiserum. The antibody is incorporated into the agar gel itself. Then the antigen is applied, diffusing into the gel and results in a circular precipitate formation. This method measures the amount of antigen.

The statistical evaluation of results was carried out using SPSS software package for epidemiological and clinical data analysis (V. 16.00, 2007 Edition).

\section{RESULTS}

Mean SIgA levels of patients in the Clinical Group and in the Control Group were $161.46 \pm 105.76$ and $69.70 \pm 25.67 \mathrm{mg}$, respectively. (Table 1) There was a statistically significant difference in the data obtained from the two groups.

Effects of gender on SigA secretion. The data processing from the Clinical Group showed a gender-related dependence of SIgA levels. SigA mean levels in female patients from the Clinical Group were significantly higher as opposed to the counterpart observations for male patients $(p=0.004)$. SigA levels in the control healthy subjects did not indicate statistically significant gender-related differences $(p=0.699)$. (Table 2).

Effects of age on SigA secretion. The effects of CKD patients' age on SigA levels were investigated through a nonparametric Mann-Whitney U Test applied to independent samples. (Fig. 1). The subjects in each group were separated into two Age Groups: 32-60 years and 61-89 years. SigA mean values for both the Clinical and Control Groups were $40.56 \pm$ 2.43 and $31.47 \pm 1.98$ years, respectively. No statistically significant correlation was detected $(p=$ 0.063). However, the data denoted a tendency as a result of the proximity to statistical significance. Further studies are to be carried out for the purpose of data refining.

The study of healthy individuals from the Control Group did not confirm association between age and SlgA levels $(p=0.985)$. 
Effects of the duration of hemodialysis treatment on SigA secretion. A statistically significant positive correlation was established between the hemodialysis duration and SIgA levels $(r=0.46, p$
$<0.001)$. The results indicated that patients who had undergone renal replacement therapy for over 5 years had significantly higher SIgA levels. (Table 3).

Table 1. SIgA mean values of patients in the Clinical Group and the Control Group

\begin{tabular}{|c|c|c|c|c|c|c|c|}
\hline Group & Number & Mean & Std. Deviation & $t$ & Sig. & Minimal value & Maximal value \\
\hline Clinical Group & 70 & 161.46 & 105.76 & \multirow{2}{*}{3.833} & \multirow{2}{*}{0.000} & 25.00 & 21.00 \\
\hline Control Group & 20 & 69.70 & 25.67 & & & 439.00 & 137.00 \\
\hline
\end{tabular}

Table 2. Gender-related differences in SIgA levels

\begin{tabular}{|l|c|c|c|c|c|c|c|}
\hline \multirow{2}{*}{ Group } & Gender & N & Mean \pm SD & SE & Range & t & p \\
\hline \multirow{2}{*}{ Clinical Group } & Men & 32 & $122.91 \pm 79.26$ & 14.01 & $25.00-284.00$ & \multirow{2}{*}{0.2 .951} & 0.004 \\
\cline { 2 - 8 } & Women & 38 & $193.92 \pm 115.01$ & 18.66 & $25.00-439.00$ & & \\
\hline \multirow{2}{*}{ Control Group } & Men & 8 & $66.87 \pm 38.02$ & 13.44 & 21.00137 .00 & -0.393 & 0.699 \\
\cline { 2 - 8 } & Women & 12 & $71.58 \pm 14.44$ & 4.17 & $55.00-100.00$ & & \\
\hline
\end{tabular}

Table 3. SIgA levels related to the duration of the renal replacement therapy

\begin{tabular}{|l|c|c|c|c|c|c|}
\hline Groups & N & Mean \pm SD & SE & Range & t & p \\
\cline { 1 - 5 } up to 5 years & 36 & $117.94 \pm 87.53$ & 14.59 & $45.00-328.00$ & \multirow{2}{*}{-3.867} & 0.000 \\
\hline over 5 years & 34 & $207.53 \pm 104.92$ & 17.99 & $25.00-439.00$ & & \\
\hline
\end{tabular}

\section{Independent-Samples Mann-Whitney U Test}

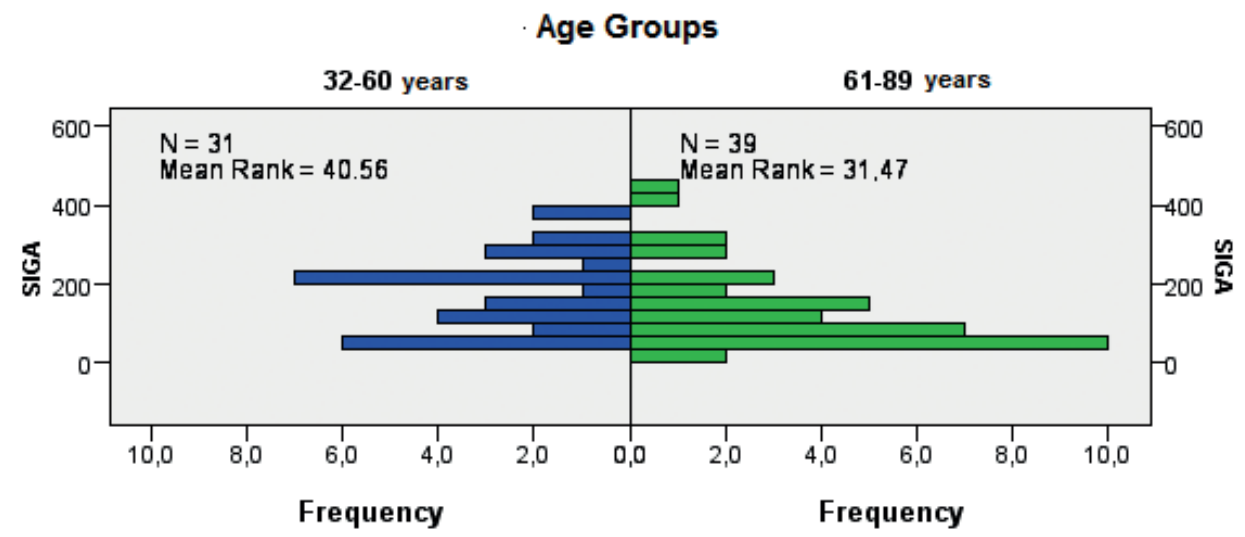

Fig. 1. Age-related mean SIgA levels in patients from the Clinical Group and the Control Group 


\section{DISCUSSION}

The standard level of immunoglobulin in saliva has not been strictly defined by researchers as its values vary depending on the origin of saliva, the method of collection and the stimulation of secretion [9]. Faris Abid Hatem et al. compared SIgA rates in 45 CKD patients and 45 control healthy subjects and established statistically significant differences. Their study revealed that the Mean \pm SD of the immunoglobulin A in chronic kidney disease patients on hemodialysis was $388.81 \pm 227.86 \mu \mathrm{g} / \mathrm{ml}$, while in control group it was $273.98 \pm 155.89 \mu \mathrm{g} / \mathrm{ml}$. The authors reported that increased concentration of salivary immunoglobulin (IgA) was usually a marker of a poor general condition [10].

In a study conducted in Finland by Vesterinen et al. SIgA concentrations were examined in CKD patients and in patients after kidney transplantation. The results revealed that the slgA concentration was highest in the hemodialysis stage, while in post-transplant stage there was a decrease in SigA values. The observation of a decrease in salivary immunoglobulin concentrations was logical and probably due to the immunosuppressant medication taken permanently after transplantation [11].

The data obtained in the present study outlined a tendency towards higher SIgA values in end-stage CKD patients compared to those in the Control Group, which matched the results of other studies $[12,13$, $14,15]$. The observations showed the effects of gender on SIgA secretion. Female patients were found to have higher SIgA levels.

SIgA values differed statistically in control healthy subjects and in end-stage CKD patients, undergoing chronic dialysis treatment. Control healthy subjects' SIgA levels were not gender - or age-related, whereas female CKD patients' SIgA was significantly increased. The studies have outlined a tendency towards increasing SIgA levels among younger pa- tients as well as in patients, undergoing chronic dialysis treatment for over 5 years.

\section{Conflicts of interest}

The authors declare no conflicts of interest.

\section{REFERENCES}

1. Bernimoulin JP. Recent concepts in plaque formation. J Clin Periodontol. 2003;30(Suppl 5): 7-90.

2. Brandtzaeg $P$, Pabst R. Let's go mucosal: communication on slippery ground. Trends Immunology. 2004; 25 (11): 570-577.

3. Bokor-Bratic M. Clinical significance of analysis of immunoglobulin A levels in saliva. Medicinskipregled. 2000; 53: 164-8.

4. Dodds MW, Jonson DA, Yeh CK. Health benefits of saliva: a review. J Dentistry. 2005; 33(3): 223-33.

5. Gonçalves TS, Morganti MA, Campos LC et al. Allergy to auto-polymerized acrylic resin in an orthodontic patient. Am J Orthod Dentofac Orthop. 2006; 129(3): 431-5.

6. Marcotte H, Lavoie MC. Oral microbial ecology and the role of salivary immunoglobulin A. Microbiol Mol Biol Rev. 1998 Mar; 62(1):71-109

7. Navazesh M, Christensen C. A comparison of whole mouth resting and stimulated salivary measurement procedures. J Dent Res, 61, 1982: 1158-1162.

8. Mancini GA, Carbonara AO, Heremans JF. Immunochemical quantitation of antigens by single radial immunodiffusion. Immunochemistry. 1965, 2.3: 235IN5-254IN6.

9. Bokor-Bratic M. Clinical significance of analysis of immunoglobulin A levels in saliva. Medicinskipregled. 2000; 53: 164-8.

10. Hatem FA, Mohammad ZJ. Salivary IgA in chronic kidney disease patients undergoing hemodialysis in Missan governorate. Journal of Baghdad College of Dentistry. 2015, 27.2: 54-57.

11. Vesterinen $\mathrm{M}$, Ruokonen $\mathrm{H}$, Furuholm $\mathrm{J}$ et al. Oral health in predialysis patients with emphasis on diabetic nephropathy. Clin Oral Investig. 2011; 15: 99-104

12. Bayraktar G, Kazancioglu R, Bozfakioglu S et al. Evaluation of salivary parameters and dental status in adult hemodialysis patients. Clin Nephrol. 2004; 62: 380-3.

13. Martin CA, Siqueira WL, De Olivira E et al. Salivary analysis of patients with chronic renal failure undergoing hemodialysis. Spec Care Dentist. 2006; 26: 205-8

14. Bayraktar G, Kurtulus I, Duraduryan A et al. Dental and periodontal findings in hemodialysis patients. Oral Dis. 2007; 13(4): 393-7.

15. Abdul-Rahman B. Salivary and plasma analysis of oxidative stress biomarkers and biochemical markers with evalution of oral manifestations in ESRD patients. A master thesis in Oral Medicine, College of Dentistry, University of Baghdad. 2011

Accepted 10.07.2019 